\title{
Vortex Collisions: Crossing or Recombination?
}

\author{
Malek Bou-Diab ${ }^{1}$, Matthew J. W. Dodgson ${ }^{1,2}$, and Gianni Blatter ${ }^{1}$ \\ ${ }^{1}$ Theoretische Physik, ETH-Hönggerberg, CH-8093 Zürich, Switzerland \\ ${ }^{2}$ Theory of Condensed Matter Group, Cavendish Laboratory, Cambridge, CB3 0HE, United Kingdom
}

(November 8, 2018)

\begin{abstract}
We investigate the collision of two vortex lines moving with viscous dynamics and driven towards each other by an applied current. Using London theory in the approach phase we observe a nontrivial vortex conformation producing anti-parallel segments; their attractive interaction triggers a violent collision. The collision region is analyzed using the time-dependent Ginzburg-Landau equation. While we find vortices will always recombine through exchange of segments, a crossing channel appears naturally through a double collision process.
\end{abstract}

PACS numbers: 74.60.Ec, 74.60.Ge

The important role that vortex collisions play in the non-equilibrium properties of type-II superconductors was recognized long ago [1,2]. E.g., vortices subject to a longitudinal (parallel to field) current are unstable towards helical expansion, leading to dissipation, but this instability is contained if the vortices cannot cut through each other. This concept goes back to Josephson [i], who assumed that the flux-line cutting process was energetically too costly to occur, a conclusion that has been rejected by Brandt et al. [3] on the basis of the first explicit calculation of the cutting energy. More recently, the extent to which entangled vortices can cut through each other has been argued [4] to determine the dynamics of the vortex-liquid phase, which dominates much of the $H-T$ plane for high- $T_{c}$ superconductors [5]. Assuming an energy barrier $U_{\times}$to vortex crossing, an exponential dependence $\tau, \eta \propto \exp \left(-U_{\times} / T\right)$ of the relaxation time $\tau$ and the viscosity $\eta$ of the vortex liquid was predicted [6,6]. Both flux transformer- and $c$-axis transport experiments [8] are particularly suitable to test these ideas.

In the original calculation of the crossing energy $U_{\times}$, Brandt et al. [3] analyzed the intersection of two straight vortices and calculated their configurational energy within London and Ginzburg-Landau theory, see also Wagenleithner [9]. Later, Sudbo and Brandt [10] found a lower barrier when all the conformal degrees of freedom of an elastic line are included: the lines bend so as to take advantage of the attraction between oppositely directed vortices. The energetics of crossing in the presence of the surrounding vortex lattice, as well as defects such as two-line and three-line twists, have been calculated, both in the London model 11] and using the lowest Landau level approximation [12]. While these previous studies have concentrated on metastable configurations, here we are concerned with the vortex scattering process itself and particularly with the output topology after the collision: vortices, being line objects, can end up in two alternative topological configurations, reswitched or crossed (see Fig. 1). In this letter we analyze the conditions selecting between these two scattering channels.
Vortex collisions have attracted a lot of interest in a variety of fields, ranging from turbulence in superfluid Helium [13] to galaxy formation via cosmic strings [14]. While the vortex dynamics is hamiltonian (Schrödinger type or massive) in these systems, the vortex dynamics in type II superconductors is dissipative in general, implying that a collision has to be driven by an external current J. Also, while the usual scattering of particles is characterized by a small number of parameters, the vortex collisions studied below involve line objects with an infinite number of degrees of freedom that eventually could influence the result of the collision. Here we study vortex collisions for a set of typical initial geometries and drives and extract the generic information at the end.

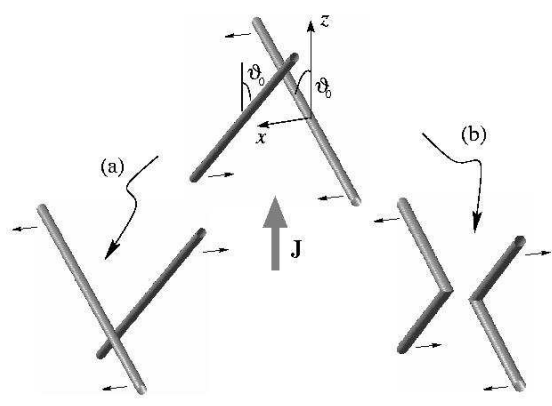

FIG. 1. Initial configuration of two colliding vortices enclosing an angle $2 \vartheta_{0}$ and pushed towards each other along the $x$-axis by the current density $\mathbf{J}$ directed parallel to the $z$-axis. The collision terminates in one of two topologically different configurations: (a) the vortices cut through each other; (b) the vortices recombine through exchange of segments.

Below, we study the collision on two different length scales: at large distances, of the order of the penetration depth $\lambda$, the vortices behave as elastic lines interacting through currents and a London description is valid. On the other hand, as the vortices approach on distances of order the coherence length $\xi$, the topology of the lines around the collision point is not well defined within the London scheme and we have to adopt the time-dependent 
Ginzburg-Landau formalism 15 (TDGL). The vortices then are described by a complex wave function which accounts for the evolution of the vortex core structure. Here, we consider the situation where these two scales are well separated, i.e., superconductors with a large Ginzburg-Landau parameter $\kappa=\lambda / \xi \sim 100$ typical for high temperature superconductors.

Our results in the London regime show that even for nearly parallel vortices and weak driving currents the two vortices always collide: the drag force acting on the vortex ends always over-compensates the local repulsive force. In addition, the induced torque twists the initially repulsive vortex segments towards a locally anti-parallel configuration with an attractive interaction, resulting in a violent collision. From our TDGL analysis at small scales we conclude that the vortices always recombine when the cores collide, independent of drive and of the angle of collision. Nevertheless, a crossing channel can appear in the system through a double collision mechanism: we find that for our geometry the vortex recombination naturally leads to an intermediate configuration which is not 'free', always enforcing a second collision. This second collision restores the original topology of the individual lines and results in a crossing configuration which is asymptotically free. These findings then lend support to an early proposal by Clem [16] for a similar double-cutting scenario for colliding vortex lines.

The initial configurations studied here are shown in Fig. 1: the two straight vortex lines $\mathcal{V}_{1}$ and $\mathcal{V}_{2}$ enclose an angle $2 \vartheta_{0}$ and are driven towards each other by an external current density $\mathbf{J}$. For large distances $d>\xi$ between the colliding vortices the system can be described within the London approximation 17,5]. The vortices are line objects parametrized by the positions $\mathbf{r}_{\mu}(z)$, where $\mu=1,2$ for the vortices $\mathcal{V}_{1}$ and $\mathcal{V}_{2}$. We concentrate on isotropic superconductors (generalization to the anisotropic case is done by rescaling [18]) and write the free energy functional in the form

$$
\begin{aligned}
\mathcal{F}_{L}\left[\left\{\mathbf{r}_{\mu}\right\}\right] & =\frac{\varepsilon_{0}}{2} \sum_{\mu, \nu=1}^{2} \int d \mathbf{r}_{\mu} \cdot d \mathbf{r}_{\nu} \frac{e^{-\sqrt{\left|\mathbf{r}_{\mu}-\mathbf{r}_{\nu}\right|^{2}+\xi^{2}} / \lambda}}{\sqrt{\left|\mathbf{r}_{\mu}-\mathbf{r}_{\nu}\right|^{2}+\xi^{2}}} \\
& +\varepsilon_{0} c_{0} \sum_{\mu=1}^{2} \int\left|d \mathbf{r}_{\mu}\right| ;
\end{aligned}
$$

the first term describes the pairwise interaction between vortex segments, with $\varepsilon_{0}=\left(\Phi_{0} / 4 \pi \lambda\right)^{2}$ the basic energy scale $\left(\Phi_{0}=h c / 2 e\right.$ is the flux unit). The terms $\mu=\nu$ correspond to the self energy of the vortices while the terms $\mu \neq \nu$ describe the interaction between them. The second part in (11) accounts for the energy of the vortex cores; the value $c_{0}=0.38$ is found from comparing the vortex energy within the London theory with the value derived from a Ginzburg-Landau analysis [19].

Below we concentrate on the vortex $\mathcal{V}_{1}$; the configuration of vortex $\mathcal{V}_{2}$ follows from symmetry. The forces acting on each point of $\mathcal{V}_{1}$ are the Lorentz force due to the current density $\mathbf{J}$, the friction force generated by the dissipation in the vortex core, and the elastic and interaction forces as given by the functional derivative of the free energy functional (i1). The dimensionless equation of motion for a point $\mathbf{r}_{1}$ on vortex $\mathcal{V}_{1}$ then takes the form

$$
\begin{aligned}
\frac{\partial \mathbf{r}_{1}}{\partial t}= & -\sum_{\mu=1}^{2} \int_{\mathcal{V}_{\mu}}\left(1+\rho_{\mu}^{-1}\right) \frac{\exp \left(-\rho_{\mu}\right)}{\rho_{\mu}^{2}} \mathbf{n}_{1} \wedge\left(\mathbf{d r}_{\mu} \wedge \mathbf{r}_{1 \mu}\right) \\
& -c_{0}\left[\mathbf{n}_{1} \wedge\left(\mathbf{n}_{1} \wedge \mathbf{k}_{1}\right)\right]+\kappa\left(\mathbf{J} \wedge \mathbf{n}_{1}\right),
\end{aligned}
$$

where $\mathbf{r}_{1 \mu}=\mathbf{r}_{1}-\mathbf{r}_{\mu}$ and $\rho_{\mu}=\sqrt{\left|\mathbf{r}_{1 \mu}\right|^{2}+1 / \kappa^{2}} ; \mathbf{n}_{1}=$ $\mathbf{r}_{1}^{\prime}(z) /\left|\mathbf{r}_{1}^{\prime}(z)\right|$ denotes the tangent vector and $\mathbf{k}_{1}=$ $\mathbf{r}_{1}^{\prime \prime}(z) /\left|\mathbf{r}_{1}^{\prime}(z)\right|^{2}\left({ }^{\prime}=d / d z\right.$ and $\left.{ }^{\prime \prime}=d^{2} / d z^{2}\right)$. Note that Eq. (2) is independent of the parameterization of the line; the forces acting on the segments are locally orthogonal. We have chosen units: $[r]=\lambda$, $[$ Force $]=\varepsilon_{0}$, $[J]=j_{0}=\varepsilon_{0} c / \xi \phi_{0}$ with $j_{0}$ of the order of the critical current density, and $[t]=\eta_{l} \lambda^{2} / \varepsilon_{0}$ with $\eta_{l}=\phi_{0}^{2} / 2 \pi \xi^{2} c^{2} \rho_{n}$ the viscosity per unit length as given by Bardeen and Stephen [17] ( $\rho_{n}$ denotes the normal state resistivity). Initially, the two vortex lines are separated by a distance $d \approx \lambda$ and enclose an angle $2 \vartheta_{0}$, see Fig. 1 . In order to describe the vortex collision including all dynamical and configurational degrees of freedom we solve the equation of motion numerically using a second-order Runge-Kutta method. The elastic forces help stabilizing the algorithm provided that the distance $\delta$ between neighboring points remains smaller than $1 / \kappa$ and the time step is $\sim \delta^{2}$.

The integration of the equation of motion for various angles $\vartheta_{0}$ and current densities $\mathbf{J}$ reveals two generic types of collisions depending on the initial angle. We first discuss the results for a 'steep' collision with a small initial angle $\vartheta_{0}<\pi / 4$, see Fig. 2. As the vortices enter the range of interaction, the vortex segments close to the $x$-axis are subject to a strong repulsive interaction. This repulsive force is compensated by the Lorentz force due to the applied current density $J$ and the drag force generated by the vortex ends. The latter is the result of the elastic forces in the line: the vortex ends far away from the center do not interact and are pushed by the Lorentz force towards the origin, their motion dragging the central part along towards collision. With decreasing separation the torque generated by the interaction grows and twists the central parts of the lines, thereby increasing the angle between the central segments. As this angle increases beyond $\pi / 2$ the interaction turns attractive, the central vortex segments turn anti-parallel and collide. After the collision these segments remain bound at a distance $d \lesssim \xi$ with both cores overlapping: the Lorentz force due to $J$ is not sufficient to separate the anti-parallel segments until the drag provided by the vortex ends has become large. Note that even a small Lorentz force (e.g., for a small angle $\vartheta_{0}$ and current drive $J$ ) is sufficient to trigger a collision due to the line twist. 
In the second, 'flat' type of collision with $\vartheta_{0}>\pi / 4$, all forces drive the vortices toward each other and they collide rapidly; the collision scenario then simplifies without the initial repulsive phase, the central vortex segments twisting smoothly and colliding violently.

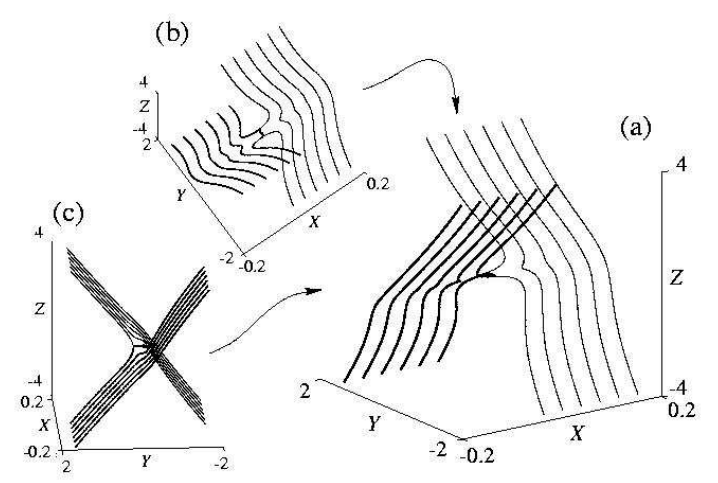

FIG. 2. Vortex configurations at constant time steps for a 'steep' collision with initial angle $\vartheta_{0}=\pi / 8$; a driving current $J=0.4 J_{0}$ has been chosen. Note the various behaviors of the central segments during the collision: repulsion, twist, attraction, and collision with binding ((a) side view, (b) top view, (c) front view, units in $\lambda$ ).

At distances $\sim \xi$ the London model is not valid; the vortex cores overlap and the interaction is dominated by the condensation rather than electromagnetic energies. We then refine the discussion of the vortex collision by solving the time-dependent Ginzburg-Landau equations [15] within a small box of size $\sim 10 \xi$ around the collision point. The vortex configuration is described by a complex wave function $\psi(\mathbf{r}, t)$ undergoing a time evolution

$$
\left(\partial_{t}-i \tilde{\phi}\right) \psi=(\nabla / \kappa+i \mathbf{A})^{2} \psi+\left(1-|\psi|^{2}\right) \psi
$$

where $\tilde{\phi}$ is the electrochemical potential and we use the units: $[r]=\lambda,[\mathbf{A}]=\sqrt{2} \lambda H_{c},[\psi]=\sqrt{|\alpha| / \beta},[t]=\tau$ $=\hbar \gamma /|\alpha|$, and $[\tilde{\phi}]=\hbar / 2 e \tau$. Here, $\alpha$ and $\beta$ are parameters in the Ginzburg-Landau free-energy, $H_{c}$ is the thermodynamic critical field, and $\gamma$ is the damping coefficient, see [15. For a clean superconductor $\gamma=1 / 2 k_{\mathrm{F}} \xi_{0}$ ( $\xi_{0}$ is the BCS coherence length) resulting in a ballistic time scale $\tau=\xi_{0} / v_{\mathrm{F}}$, while in the dirty case $\gamma=3 / 2 k_{\mathrm{F}} l$ ( $l$ the mean free path) with a diffusive time $\tau=3 \xi_{0}^{2} / l v_{\mathrm{F}}\left(k_{\mathrm{F}}\right.$ and $v_{\mathrm{F}}$ denote the Fermi wave vector and Fermi velocity). Eq. (3) is completed by the Maxwell equations and the constitutive relations for the superconductor [15], of which we need the expressions $\mathbf{j}_{n}=-2 \Sigma\left(\partial_{t} \mathbf{A}+\nabla \phi / \kappa\right)$ and $\mathbf{j}_{s}=i\left[\psi^{*}(\nabla / \kappa+i \mathbf{A}) \psi-\right.$ c.c. $]$ for the normal and superconducting current densities with $\Sigma=m \beta / 4 e^{2} \hbar \rho_{n} \gamma$; the continuity equation $\partial_{t} \rho+\nabla \cdot\left(\mathbf{j}_{n}+\mathbf{j}_{s}\right)=0$ with $\rho$ the charge density can then be used to determine the potential $\tilde{\phi}$. Using the values of $\beta$ and $\gamma$ derived from microscopic theory 15 the coefficient $\Sigma$ takes the value $\Sigma=14 \zeta(3) / \pi^{4}$ in the dirty case, while $\Sigma=4 l / \pi^{3} e^{-C} \xi_{0}$ for a clean superconductor ( $C$ is the Euler constant); below we concentrate on the parameter-free dirty situation. As we are interested in the core region (i.e., scales smaller than $\lambda$ ) we may ignore transverse screening by choosing a gauge with $\mathbf{A}=0$. Furthermore, neglecting weak charging effects [15] the requirement of divergence free flow,

$$
\Sigma \nabla^{2} \tilde{\phi}=(i / 2)\left[\psi^{*} \nabla^{2} \psi-\psi \nabla^{2} \psi^{*}\right]
$$

determines the electrochemical potential $\tilde{\phi}$ needed to push the normal current density $\mathbf{j}_{n}$ through the vortex core. We use the initial condition

$$
\psi(\mathbf{r}, t=0)=\psi_{1} \psi_{2} \sqrt{1-\nu^{2}} \exp (-i \kappa \nu z)
$$

where $\psi_{i}, i=1,2$ denote the wave functions for the vortices $\mathcal{V}_{i}\left[\psi(r, \varphi)=\left(\kappa r / \sqrt{\kappa^{2} r^{2}+2}\right) \exp i \varphi\right]$ appropriately rotated and translated. The last factor in (5) describes the driving current $j_{s}=2 \nu\left(1-\nu^{2}\right)$ along the $z$-axis with a tuning parameter $\nu$ and a prefactor that takes into account the reduction in the superfluid density due to the applied current. This applied current is maintained during the time evolution through the boundary conditions $\partial_{z} \psi=-i \kappa \nu \psi$ on the top and bottom of the box. We always adapt the dimensions of the box such that the cores penetrate through the sides in order not to disturb the applied current density; we use two types of boundary conditions on the box sides to make sure that our results are not influenced by our specific choice: $i$ ) the boundary condition $\nabla_{\perp} \psi=0$ guarantees that no currents leave the box through the sides, and $i i)$ we impose the boundary condition $\psi(\mathbf{r}, t)=\psi_{1}(\mathbf{r}-\mathbf{v} t) \psi_{2}(\mathbf{r}+\mathbf{v} t) \sqrt{1-\nu^{2}} \exp (-i \kappa \nu z)$ (c.f. (5)) with the velocity $\mathbf{v}$ adapted to the local velocity of the vortex close to the boundary. We start with the vortices separated a distance of a few $\xi$ in order to have the wave functions relax before they enter the collision region and integrate Eq. (3) using the Forward-Euler method which converges well for our dissipative dynamics. The Poisson equation (4) for $\tilde{\Phi}(\mathbf{r}, t)$ is integrated at each time step using a conjugate gradient method [20].

A systematic survey for different angles and applied current densities smaller than the depairing current provides the following results: any collision, independent of initial angle or drive, makes the vortices recombine through exchange of segments, see snapshots (a) to (c) in Fig. 3. The resulting reswitched configuration is not 'asymptotically free': the drive current now acts differently on the newly built vortex lines and pushes the two vortex ends of each line into opposite directions, inducing a twist of the central vortex segments. As these segments turn around, the Lorentz force changes direction and the vortices are driven towards a new collision (snapshot (d)). The second collision (snapshot (e)) then enforces another recombination, resulting in a 'crossing' topology as if the original colliding vortices had cut through each other (snapshot (f); we have found this double collision to 
occur in all our simulations with $\vartheta_{0} / \pi \in[1 / 12,5 / 12]$ and $\left.j / j_{0} \in[0.15,0.75]\right)$. We thus find a rather unexpected result: while each (local) collision induces a change in topology through recombination, double collisions occur naturally due to geometrical constraints and lead to vortex crossing - the asymptotic regime where the vortices can separate is only reached when the vortices have crossed after a second collision.
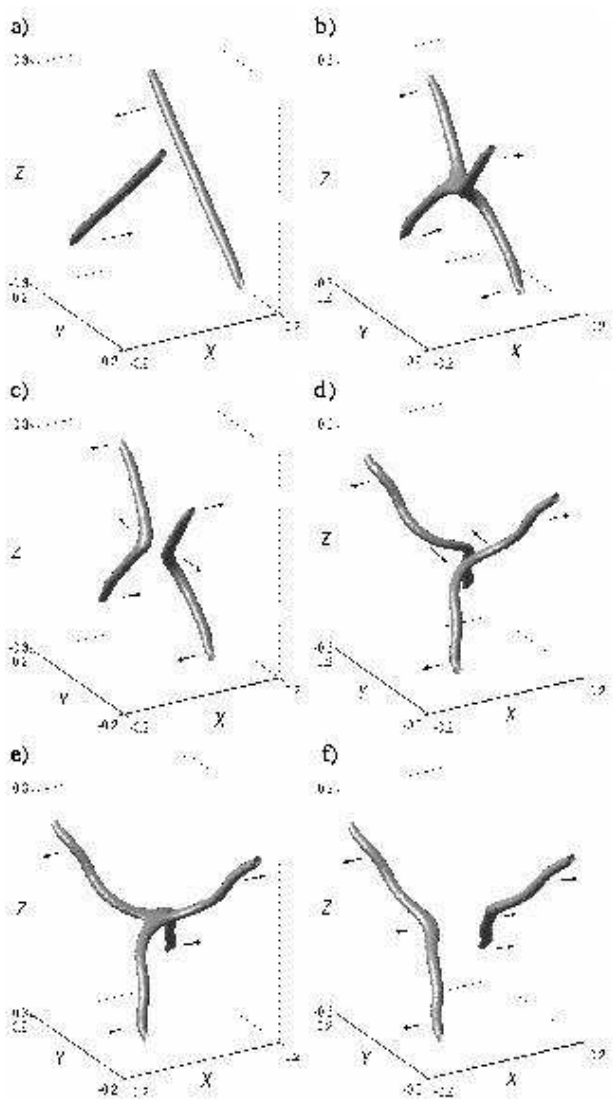

FIG. 3. Time evolution of a vortex collision calculated within TDGL theory for $\vartheta_{0}=\pi / 4$ and $J=0.6 J_{0}$. The time sequence (from left to right and top to bottom) reveals a double collision process: (a)-(c) first collision with switched segments due to recombination; (d) central segments twist and prepare for second collision (e); (f) second recombination with asymptotically free vortices in the crossing topology.

While we have studied the generic situation of two individual colliding vortices, vortex collisions occur and are important in other situations, e.g., within the vortex solid or liquid phase of (high- $T_{c}$ ) superconductors. The 'asymptotic' regimes defining the initial and final states of the collision may then look quite different and the second collision producing vortex crossing, which is generic to the above geometry, may be absent. An example for such a situation is the expansion of a vortex twist 11 in a vortex lattice or liquid phase. The interaction with neighboring vortices inhibits the second twist of the segments and each collision terminates after one encounter - as a result, the vortex twist (or ring) smoothly expands through recombination processes. Such recombination processes have also been suggested to relax the stress in driven vortex systems [7]. Furthermore, vortex recombination has been assumed to help relax the pitch of spiral vortices in current carrying superconducting cylinders subjected to a longitudinal field 21.

Summarizing, colliding vortices in type-II superconductors always recombine through exchange of segments. However, the line nature of the vortices can enforce a second collision, opening a vortex crossing channel through a double collision. The two cases are easily distinguished through careful inspection of the asymptotic constraints enforced on the collision.

We thank V. Geshkenbein, M. Troyer, and M. Cates for stimulating discussions, and the Swiss National Foundation for financial support.

[1] B.D. Josephson, Phys. Rev. 152, 211 (1966).

[2] A. Campbell and J. Evetts, Adv. Phys. 21, 199 (1972).

[3] E.H. Brandt, J.R. Clem, and D.G. Walmsley, J. Low Temp. Phys. 37, 43 (1979).

[4] M.C. Marchetti and D.R. Nelson, Phys. Rev. B 42, 9938 (1990).

[5] G. Blatter et al., Rev. Mod. Phys. 66, 1125 (1994).

[6] S.P. Obukhov and M. Rubinstein, Phys. Rev. Lett. 66, 2279 (1991).

[7] M.E. Cates, Phys. Rev. B 45, 415 (1992).

[8] R. Busch et al., Phys. Rev. Lett. 69, 522 (1992); H. Safar et al., Phys. Rev. Lett. 72, 1272 (1994); D. Lopez et al., Phys. Rev. Lett. 76, 4034 (1996) and 80, 1070 (1998).

[9] P. Wagenleithner, J. Low Temp. Phys. 48, 25 (1982).

[10] A. Sudbo and E.H. Brandt, Phys. Rev. Lett. 67, 3176 (1991).

[11] A. Schönenberger, V. Geshkenbein, and G. Blatter, Phys. Rev. Lett. 75, 1380 (1995).

[12] M.J.W. Dodgson and M. Moore, Phys. Rev. B 51, 887 (1995); M. Moore and N. Wilkin, Phys. Rev. B 50, 294 (1994).

[13] K.W. Schwarz, Phys. Rev. B 31, 5782 (1985); 38, 2398 (1988); J. Koplik and H. Levine, Phys. Rev. Lett. 71, 1375 (1993).

[14] E.P.S. Shellard, Nuclear Physics B283, 624 (1987); P. Laguna and R.A. Matzner, Phys. Rev. D 411751 (1990).

[15] A. Schmid, Phys. Kondens. Materie 5, 302 (1966).

[16] J.R. Clem, Physica 107B, 453 (1981).

[17] M. Tinkham, Introduction to Superconductivity (McGraw Hill, Inc., New York, 1996).

[18] G. Blatter, V.B. Geshkenbein, and A.I. Larkin, Phys. Rev. Lett. 68, 875 (1992).

[19] C.R. Hu, Phys. Rev. B 6, 1756 (1972).

[20] Numerical implementation was simplified by the Blitz++ library, see T.L. Veldhuizen, Lecture Notes in Computer Science 1505, 223 (1998).

[21] J.R. Clem, Phys. Rev. Lett. 38, 1425 (1977). 